\title{
Magnon damping in ferromagnetic semiconductors under intense radiation and magnetic fields
}

\author{
P. R. R. Pereira and L. C. M. Miranda* \\ Departamento de Física, Universidade de Brasilia, 70.000 Brasilia, D.F. Brazil
}

(Received 6 August 1976)

\begin{abstract}
The magnon scattering by electrons in the simultaneous presence of radiation and magnetic fields is discussed. A kinetic equation is derived, and the rate of change of the magnon population is calculated. For circularly polarized radiation propagating parallel to the magnetic field it is found that multiphoton processes are dominant when the radiation frequency is near the electron cyclotron frequency. Furthermore, the damping rate is found to decrease exponentially as the radiation frequency approaches the electron cyclotron frequency.
\end{abstract}

In a recent paper ${ }^{1}$ we reported on the modification of the spin-wave damping in an electronmagnon system in the presence of a classical electromagnetic wave. The electron states were described by the exact solution to the time-dependent Schrödinger equation for an electron in the field of classical electromagnetic wave, within the dipole approximation, without taking into account the presence of a dc magnetic field. This is justifiable provided the frequency $\omega$ of the radiation field is much greater than the electron cyclotron frequency $\omega_{c}=e B / m c$. As expected, external fields which change the spectrum and the occupation numbers of the electron states will influence the spectrum and damping of the spin waves. In fact, it was found in Ref. 1 that, in the strong-field limit, the electron-magnon collision involving multiphoton absorption becomes dominant which, in turn, entails that the magnon population may become unstable (grow with time).

Although the laser frequency is usually much greater than the electron cyclotron frequency, a resonance condition, where the laser frequency is equal to the cyclotron frequency, may be approached either by increasing the magnetic field strength or by using longer-wavelength lasers. Since intense submillimeter lasers are becoming available ${ }^{2}$ it is therefore important to consider the effect of the cyclotron resonance absorption of this radiation on the electron-magnon scattering.

Hence, in the present paper we extend the theory of Ref. 1 by considering the magnon scattering by electrons in the field of a laser beam and include the effects of an external uniform magnetic field. Our model for a magnetic semiconductor is that of an interacting conduction-electron-localizedmoment system. ${ }^{3-5}$ We describe the electron system by a parabolic conduction band and the local moment part by interacting (direct exchange) ionic moments $g \mu_{B} \vec{S}_{i}$ localized at lattice sites $\vec{R}_{i}$. The carriers and the localized moments are in- teracting by their exchange interaction which is taken to have the familiar $s-d$ contact form. The electron states are described by the solution to the Schrödinger equation for an electron in the simultaneous presence of a classical electromagnetic wave and a uniform magnetic field. The magnon scattering by the electrons is treated using first-order perturbation theory, however, retaining the radiation field strength to all orders. The transition probabilities are then used to write a kinetic equation for the magnon population from which the damping rate is obtained. For the case of a right-hand circularly polarized plane wave propagating parallel to the magnetic field (assumed to be along the $+z$ direction), the damping rate is found to decrease as the laser frequency approaches the electron cyclotron frequency. A simple physical explanation of this result is also given.

The solution to the time-dependent Schrödinger equation for an electron in the presence of a righthand circularly polarized plane wave propagating parallel to a uniform magnetic field ( $z$ axis) is given in Refs. 6 and 7. In the case of a ferromagnetic semiconductor, however, the conduction band becomes spin split ${ }^{3-5}$ such that one adds to the free-carrier energy the term $-\frac{1}{2}\left(\hbar \omega_{c}+J S\right) \sigma$, where $\sigma=+1$ for up carrier moments and $\sigma=-1$ for down moments. Here $J$ is the $s-d$ exchange parameter between the localized spins $S$ and the conduction electrons. Since $J S$ is usually much greater than $\hbar \omega_{c}$, we shall in the following approximate the band splitting by $-\frac{1}{2} J S \sigma$. Hence, including the band splitting, the electron wave function in a magnetic semiconductor in the simultaneous presence of an electromagnetic wave and a dc magnetic field is given $b^{6,7}$

$$
\begin{aligned}
\psi_{\alpha \sigma}(\overrightarrow{\mathrm{x}}, t)= & L^{-1} \exp \left(\frac{i \overrightarrow{\mathrm{p}} \cdot \overrightarrow{\mathrm{x}}}{n}\right) \exp \left(-i\left(E_{n}-\frac{1}{2} J S \sigma\right) \frac{t}{\hbar}\right) \\
& \times \exp \left(-\frac{i}{2 m \hbar} \int^{t} d t^{\prime} R\left(t^{\prime}\right)\right) \phi_{n}(\zeta),
\end{aligned}
$$


where

$$
\begin{aligned}
& E_{n}=\hbar \omega_{c}\left(n+\frac{1}{2}\right), \quad n=0,1,2, \ldots, \\
& \overrightarrow{\mathrm{p}}=\left(p_{x}, Q(t), p_{z}\right), \\
& R(t)=[\overrightarrow{\mathrm{p}}-(e / c) \overrightarrow{\mathrm{A}}(t)]^{2}-\left[p_{x}-G(t)\right]^{2}, \\
& \phi_{n}(\zeta)=\left(m \omega_{c} / \pi \hbar\right)^{1 / 4}\left(2^{n} n !\right)^{-1 / 2} \exp \left(-\frac{1}{2} \zeta^{2} H_{n}(\zeta)\right), \\
& \zeta=\left(m \omega_{c} / \hbar\right)^{1 / 2} y-\left(m \omega_{c} \hbar\right)^{-1 / 2}\left[p_{x}-G(t)\right] .
\end{aligned}
$$

Here $p_{x}$ and $p_{z}$ are constants of motion, $n$ is the Landau-level quantum number, and $\phi_{n}$ is the harmonic-oscillator wave function. The real functions $Q(t)$ and $G(t)$ are determined by

$$
\begin{aligned}
G(t)+i Q(t)=\frac{e \omega_{c}}{c} \int^{t} d t^{\prime} \\
\\
\times\left[A_{y}\left(t^{\prime}\right)-i A_{x}\left(t^{\prime}\right)\right] \exp \left[i \omega_{c}\left(t^{\prime}-t\right)\right],
\end{aligned}
$$

where $A_{x}(t)$ and $A_{y}(t)$ are the components of

$$
\overrightarrow{\mathrm{A}}(t)=\left(c E_{0} / \omega\right)\left(\overrightarrow{\mathrm{e}}_{x} \cos \omega t+\overrightarrow{\mathrm{e}}_{y} \sin \omega t\right) \text {. }
$$

We refer to Refs. 6 and 7 for a more detailed discussion of the above solution.

Treating the electron-magnon interaction as a perturbation, the probability amplitude for the transition from state $\left(\alpha_{1}=n_{1}, p_{1 x}, p_{1 z} ; \sigma_{1}=+1\right)$ to state $2\left(\alpha_{2}=n_{2}, p_{2 x}, p_{2 z} ; \sigma_{2}=-1\right)$ due to the collision with a magnon of momentum $\hbar \overrightarrow{\mathrm{k}}$ is $^{3-5}$

$$
\begin{aligned}
a(1-2 ; \overrightarrow{\mathrm{k}})=-\frac{i}{\hbar} \iint & d^{3} x d t \\
& \times \psi_{\alpha_{2 \downarrow}}^{*}(\overrightarrow{\mathrm{x}}, t) M_{s d} e^{i\left(\overrightarrow{\mathrm{k}} \cdot \overrightarrow{\mathrm{x}}-\omega_{k} t\right)} \psi_{\alpha_{1 \dagger} \dagger}(\overrightarrow{\mathrm{x}}, t),
\end{aligned}
$$

where $M_{s d}=-J(S / 2 N)^{1 / 2}$ is the $s-d$ electron-magnon coupling, with $N$ being the number of localized spins in the crystal of volume $V=L^{3}$. Substituting Eq. (1) into Eq. (4) and proceeding as in Refs. 1 and 7 , the transition probability per unit time, $T_{\nu}(\alpha \uparrow \rightarrow \beta \downarrow ; \overrightarrow{\mathrm{k}})$, for the transition from the $\alpha \uparrow$ to state $\beta \downarrow$ due to a collision with a magnon $\vec{k}$ with absorption $(\nu>0)$ or emission $(\nu<0)$ of $|\nu|$ photons may be written

$$
\begin{aligned}
T_{\nu}(\alpha \rightarrow \beta ; \overrightarrow{\mathrm{k}})= & (2 \pi / \hbar) M_{s d}^{2} J_{\nu}^{2}(\lambda / \hbar \omega)|\chi(n+l, n, \rho)|^{2} \\
& \times \delta\left(E_{\beta \downarrow}-E_{\alpha \uparrow}-\hbar \omega_{k}-\nu \hbar \omega\right)
\end{aligned}
$$

where

$$
\begin{aligned}
& E_{B \downarrow}=\hbar \omega_{c}\left(n+l+\frac{1}{2}\right)+\left(p_{z}+\hbar k_{z}\right)^{2} / 2 m+\frac{1}{2} J S, \\
& E_{\alpha \uparrow}=\hbar \omega_{c}\left(n+\frac{1}{2}\right)+p_{z}^{2} / 2 m-\frac{1}{2} J S,
\end{aligned}
$$

$J_{\nu}$ is the Bessel function of order $\nu$,

$$
\lambda=e \hbar k E_{0} / m\left(\omega-\omega_{c}\right)=\hbar k v_{0}
$$

is the field parameter, $\rho=\hbar k^{2} / 2 m \omega_{c}$, and $\chi(n+l, n, \rho)$ is the overlap of the Landau harmonic oscillators as defined, for instance, in Ref. 8.

The rate of change of the number of magnons of wave number $k, d N_{k} / d t$, is then given in terms of the transition probability as ${ }^{1}$

$$
\frac{d N_{k}}{d t}=\gamma_{k} N_{k}
$$

where

$$
\begin{aligned}
\gamma_{k}=\sum_{n p_{x} p_{z}} \sum_{i=-\infty}^{+\infty} \sum_{\nu=-\infty}^{+\infty} & \frac{2 \pi}{\hbar} M_{s d}^{2}|\chi(n+l, n, \rho)|^{2} J_{\nu}^{2}\left(\frac{\lambda}{\hbar \omega}\right) \\
& \times\left[f\left(E_{B \downarrow}\right)-f\left(E_{\alpha \uparrow}\right)\right] \\
& \times \delta\left(E_{B \downarrow}-E_{\alpha \uparrow}-\hbar \omega_{k}-\nu \hbar \omega\right) .
\end{aligned}
$$

In the following we assume a Maxwellian distribution and consider only the cyclotron resonance case, namely $\omega \sim \omega_{c}$. Then $\lambda \gg \hbar \omega$ and the argument of the Bessel function is large. For large values of the argument, the Bessel function is small except when the order $\nu$ is equal to the argument. The sum over $\nu$ in Eq. (7) may then be written approximately as

$$
\sum_{\nu=-\infty}^{+\infty} J_{\nu}^{2} \frac{\lambda}{\hbar \omega} \delta(E-\nu \hbar \omega) \simeq \frac{1}{2}[\delta(E-\lambda)+\delta(E+\lambda)] .
$$

The factor $\frac{1}{2}$ may be verified by integrating both sides of the equation over $E=E_{\beta_{\downarrow}}-E_{\alpha \uparrow}-\hbar \omega_{k}$. The first $\delta$ function corresponds to the emission and the second to the absorption of $\lambda / \hbar \omega$ photons. Since $\lambda \gg \hbar \omega$, only multiphoton processes are significant. The magnon damping then becomes

$$
\begin{aligned}
\gamma_{k}=\frac{\pi}{\hbar} \sum_{n p_{x} p_{z}} \sum_{l=-\infty}^{+\infty} M_{s d}^{2}|\chi(n+l, n, \rho)|^{2}[ & f\left(E_{\alpha \uparrow}\right)\left(e^{-\left(\lambda+\hbar \omega_{k}\right) / k_{B} T}-1\right) \\
& \left.\times \delta\left(E_{B \downarrow}-E_{\alpha \uparrow}-\hbar \omega_{k}-\lambda\right)+f\left(E_{\alpha \uparrow}\right)\left(e^{\left(\lambda-\hbar \omega_{k} / k_{B} T\right.}-1\right) \delta\left(E_{B \downarrow}-E_{\alpha \uparrow}-\hbar \omega_{k}+\lambda\right)\right] .
\end{aligned}
$$

Furthermore, assuming that $\lambda \gg k_{B} T$ for $\omega$ near $\omega_{c}$, the contribution of processes in which photons are emitted is negligible compared to the contribution of processes in which processes are absorbed. Under these circumstances Eq. (8) becomes

$$
\begin{aligned}
\gamma_{k}=\frac{\pi}{\hbar} \sum_{l=-\infty}^{+\infty} \sum_{n, p_{x}, p_{z}} & M_{s d}^{2}|\chi(n+l, n, \rho)|^{2} f\left(E_{\alpha \uparrow}\right) \\
& \times\left(e^{\left(\lambda-\hbar_{\omega_{k}}\right) k_{B} T}-1\right) \\
& \times \delta\left(E_{\beta_{l}}-E_{\alpha \uparrow}-\hbar \omega_{k}+\lambda\right) .
\end{aligned}
$$


Equation (9) is quite general and valid for any radiation and magnetic field strengths. The only assumptions are that $\omega$ is near $\omega_{c}$ and that the electrons obey a Maxwellian distribution. Since the existence in most ferromagnetic semiconductors of well defined Landau levels $\left(\hbar \omega_{c} \gg k_{B} T\right.$ and $\left.\omega_{c} \tau>1\right)$ is not easily attainable with relatively low magnetic fields, ${ }^{8}$ we shall in the following consider the electron motion in each spin-split band classically (i.e., low $B$ and large $n$ ). Accordingly we take the classical limit of Eq. (9) by formally letting

$$
\hbar \rightarrow 0 \text { and } n \rightarrow \infty,
$$

such that

$$
n \hbar \omega_{c} \rightarrow \frac{1}{2} m v_{\perp}^{2}
$$

and

$$
\sum_{n, p_{x}, p_{z}}(\cdots) f\left(E_{n p_{x} p_{z}}\right)-V \int d^{3} v(\vec{v})(\cdots) .
$$

Hence, expanding Eq. (9) in powers of $\hbar$ and retaining only the lowest-order terms, one gets

$$
\begin{aligned}
\gamma_{k}=\frac{\pi V M_{s d}^{2}}{\hbar k_{B} T}\left(k v_{0}-\omega_{k}\right) \\
\quad \times \sum_{l=-\infty}^{+\infty} \int d^{3} v J_{l}^{2}\left(\frac{k_{\perp} v_{\perp}}{\omega_{c}}\right) f_{\uparrow}(\overrightarrow{\mathrm{v}}) \\
\quad \times \delta\left(l \omega_{c}+k_{z} v_{z}+J S-\omega_{k}+k_{\perp} v_{0}\right),
\end{aligned}
$$

where we have written $\lambda$ as $\hbar k_{\perp} v_{0}$ with $v_{0}$ $=e E_{0} / m\left|\omega-\omega_{c}\right|$, and replaced $|\chi(n+l, n, \rho)|^{2}$ by its classical limit, ${ }^{9}$ namely

$$
|\chi(n+l, n, \rho)|^{2} \rightarrow J_{l}^{2}\left(k_{\perp} v_{\perp} / \omega_{c}\right) \text {. }
$$

For $E_{0}=0\left(v_{0}=0\right)$, it can be shown that Eq. (13) reduces to the expression of the magnon damping in a uniform magnetic field ${ }^{8}$ in the classical limit. On the other hand, for $B \rightarrow 0$ the argument of the Bessel functions in Eq. (13) is large so that, as before, we may approximate $\gamma_{k}$ by

$$
\begin{aligned}
\gamma_{k} \underset{B \rightarrow 0}{\rightarrow} & \frac{\pi V M_{s d}^{2}}{\hbar k_{B} T}\left(k_{\perp} v_{0}-\omega_{k}\right) \\
& \times \int d^{3} v f_{\uparrow}(\overrightarrow{\mathrm{v}}) \delta\left(\overrightarrow{\mathrm{k}} \cdot \overrightarrow{\mathrm{v}}-\omega_{k}+k_{\perp} v_{0}+J S\right) .
\end{aligned}
$$

This is exactly the same result as the one reported in the previous paper ${ }^{1}$ for the case of nondegenerate semiconductors in the limit of the $E_{0}$ large, Finally, for $E_{0}=0$, Eq. (14) reproduces the results of Ref. 10.

Going back to Eq. (13), substituting $f_{\uparrow}(\overrightarrow{\mathrm{v}})$ by ${ }^{10}$

$$
\frac{1}{2} n_{0}\left[\boldsymbol{e}^{J S / 2 k_{B} T} / \cosh \left(J S / 2 k_{B} T\right)\right]\left(\pi_{T}^{2}\right)^{-3 / 2} e^{-v^{2} / v_{T}^{2}},
$$

where $v_{T}^{2}=2 k_{B} T / m$, and performing the integration over $v_{z}$ using the $\delta$ function, one has

$$
\gamma_{k}=\frac{\pi^{1 / 2} n_{0} V M_{s d}^{2}}{\hbar m v_{T}^{2}} \frac{\exp \left(J S / 2 k_{B} T\right)}{\cosh \left(J S / 2 k_{B} T\right)}\left(\frac{k_{\perp} v_{0}-\omega_{k}}{\left|k_{z}\right| v_{T}}\right) \sum_{l=-\infty}^{+\infty} \exp \left(\frac{-\left(k_{\perp} v_{0}+l \omega_{c}+J S-\omega_{k}\right)^{2}}{k_{z}^{2} v_{T}^{2}}\right) F_{l}\left(\frac{k_{\perp} v_{T}}{\omega_{c}}\right)
$$

where

$$
\begin{aligned}
F_{l}\left(\frac{k_{\perp} v_{T}}{\omega_{c}}\right) & =\int_{0}^{\infty} d x e^{-x} J_{l}^{2} \frac{k_{\perp} v_{T}(x)^{1 / 2}}{\omega_{c}} \\
& =\exp \left(-k_{\perp}^{2} v_{T}^{2} / 2 \omega_{c}^{2}\right) I_{l}\left(k_{\perp}^{2} v_{T}^{2} / 2 \omega_{c}^{2}\right) .
\end{aligned}
$$

It follows from Eq. (15) that, regardless of the value of $E_{0}$, for $\omega=\omega_{c}\left(v_{0} \rightarrow \infty\right) \gamma_{k}$ vanishes. Physically this result may be understood as follows. Consider the problem of one electron in an electromagnetic field described by $\overrightarrow{\mathrm{A}}(t)$ and moving in a potential $V$ (the localized moment potential). We have

$$
H=\frac{1}{2} m v_{z}^{2}+\frac{1}{2} m v_{0}^{2}+V,
$$

where $\frac{1}{2} m v_{\varepsilon}^{2}$ and $\frac{1}{2} m v_{0}^{2}$ are the longitudinal and transverse energies of the electron, respectively. Then for $\omega=\omega_{c}$, the transverse energy is much larger than $V$, so that $H$ may be approximated by the free-electron Hamiltonian $H_{0}=\frac{1}{2} m v^{2}$. In other words, at $\omega=\omega_{c}$ the electron no longer sees the

potential $V$.

The second point regarding Eq. (15) is that for $\omega$ near $\omega_{c}$, but not necessarily at resonance, the magnon population may, in principle, grow with time for wave vectors $\overrightarrow{\mathrm{k}}$ in a general direction 0 , provided $k_{\perp} v_{0}>\omega_{k}$. This condition is amply satisfied for $\omega \sim \omega_{c}$. However, the estimative of the growth rate from Eq. (15) is quite tedious. Nevertheless, one may get the order of magnitude of the size of this effect by considering the case where $k_{\perp} v_{T} \ll \omega_{c}$. In this case, the $l=0$ component in Eq. (15) is the dominant one, and we have

$$
\begin{aligned}
\gamma_{k}= & \frac{\pi^{1 / 2} n_{0} V M_{s d}^{2}}{\hbar m v_{T}^{2}} \frac{\exp \left(J S / 2 k_{B} T\right)}{\cosh \left(J S / 2 k_{B} T\right.} \frac{\left(k_{\perp} v_{0}-\omega_{k}\right)}{\left|k_{z}\right| v_{T}} \\
& \times \exp \left(\frac{-\left(k_{\perp} v_{0}+\omega_{s}-\omega_{k}\right)^{2}}{K_{z}^{2} v_{T}^{2}}\right),
\end{aligned}
$$

which is formally analogous to the result of Ref. 
10 for the magnon damping rate due to the $s-d$ scattering in the presence of a dc electric field. The difference is that in Ref. $10 v_{0}$ is replaced by $v_{d}$ (the electron drift velocity).

So far we have completely ignored the collisional damping of the electron states which actually may prevent the divergence of $\lambda_{\kappa}$ at $\omega=\omega_{c}$ (i.e., we have assumed $\left.\omega_{c} \tau \rightarrow \infty\right)$. Hence in applying the above results to actual physical systems some caution should be exercised. The fact that for most magnetic semiconductors $\omega_{c} \tau$ is not infinitely large means that one should restrict ourselves to the case of high-mobility materials. Of the existing ferromagnetic semiconductors the best candidate ${ }^{11-13}$ is $\mathrm{Ag}$-doped $\mathrm{CdCr}_{2} \mathrm{Se}_{4}\left(T_{c}=130{ }^{\circ} \mathrm{K}, \mu \simeq 10^{4}\right.$ $\mathrm{cm}^{2} / \mathrm{V} \mathrm{sec}$ at $T=120{ }^{\circ} \mathrm{K}$ ) for which $\omega_{c} \tau \sim 4(10)$ for $H=40(100) \mathrm{kOe}$. One notices that for $T=120^{\circ} \mathrm{K}$ and $H$ between 40 and $100 \mathrm{kOe} . K_{B} T \gg \hbar \omega_{c}$ so that the assumption of large $n$ is still valid. Now, if $\omega_{c} \tau \sim 1$, then at resonance $\left|\omega-\omega_{c}\right|$ is typically of the order of $1 / \tau$ with the result that $\lambda / \hbar \omega_{c}$ will approximately be given by $\mu k_{\perp} E_{0} / \omega_{c}$.

In this case, condition $\lambda / \hbar \omega_{c} \gg 1$, and therefore the validity of our results [Eqs. (9)-(16)], is achieved not by a frequency matching but rather by increasing the radiation field strength $E_{0}$. Since at microwave frequencies field strengths up to $10^{4} \mathrm{~V} / \mathrm{cm}$ can be obtained using pulsed techniques, conditions $\lambda / \hbar \omega_{c} \gg 1$ and $k_{\perp} v_{0}>\omega_{k}$ can be achieved. For instance, for $H=40 \mathrm{kOe}, k=10^{6}$ $\mathrm{cm}^{-1}$, and $\mu \sim 10^{4} \mathrm{~cm}^{2} / \mathrm{V} \mathrm{sec}$, both conditions $\lambda / \hbar \omega_{c} \gg 1$ and $k_{\perp} v_{0}>\omega_{k}$ are satisfied for $E_{0} \sim 800$ $\mathrm{V} / \mathrm{cm}$.

Although our model contains a number of simplifying assumptions we believe that, at least qualitatively, the essential conclusions may be useful in explaining future cyclotron-resonance case of high-mobility materials (e.g., Ag-doped $\left.\mathrm{CdCr}_{2} \mathrm{Se}_{4}\right)$. A weakening of the electron-magnon interaction as $\omega$ approaches $\omega_{c}$ is predicted. This weakening becomes further evident by increasing the radiation field strength.
*Present address: Instituto de Fisica, UNICAMP, C. P. $1170,13.100$ Campinas, SP, Brazil.

${ }^{1}$ S. Coutinho and L. C. M. Miranda, Phys. Rev. B (to be published).

${ }^{2}$ B. Lax and D. R. Cohn, Appl. Phys. Lett. $\underline{23}, 363$ (1973).

${ }^{3}$ R. B. Woolsey and R. M. White, Phys. Rev. B 1,4474 (1970).

${ }^{4}$ S. Methfessel and D. C. Mattis, in Handbuck der Physik, edited by S. Flügge (Springer, Berlin, 1962), Vol. XVIII/I.

${ }^{5}$ S. V. Vonsovski and Yu. A. Yzyumov, Usp. Fiz. Nauk. 78, 3 (1963) [Sov. Phys-Usp. 5, 723 (1963)].
${ }^{6}$ J. F. Seely, Am. J. Phys. 42, 326 (1974).

${ }^{7}$ J. F. Seely, Phys. Rev. A $\overline{10}, 1863$ (1974).

${ }^{8}$ M. A. F. Gomes and L. C. $\bar{M}$. Miranda, Phys. Rev. B 12, 3788 (1975).

${ }^{9}$ G. $\bar{M}$. Walters and E. G. Harris, Phys. Fluids 11,112 (1965).

${ }^{10}$ M. D.Coutinho, Jr., L. C. M. Miranda, and S. M. Rezende, Phys. Status Solidi B 65, 689 (1974).

${ }^{11}$ I. Balberg and H. L. Pinch, Phys. Rev. Lett. 28, 909 (1972).

${ }^{12}$ H. W. Lehmann, Phys.Rev. 163, 488 (1967).

${ }^{13}$ C. Haas, Phys. Rev. 168, $5 \overline{31}$ (1967). 